\title{
Off-Label Use of Letermovir as Preemptive Anti-Cytomegalovirus Therapy in a Pediatric Allogeneic Peripheral Blood Stem Cell Transplant
}

This article was published in the following Dove Press journal: Infection and Drug Resistance

\author{
Angela Chiereghin $\mathbb{D}^{1,2}$ \\ Tamara Belotti ${ }^{3}$ \\ Eva Caterina Borgatti ${ }^{4}$ \\ Nicola Fraccascia ${ }^{5}$ \\ Giulia Piccirilli ${ }^{6}$ \\ Maura Fois ${ }^{3}$ \\ Michele Borghi ${ }^{4}$ \\ Gabriele Turello ${ }^{4}$ \\ Liliana Gabrielli ${ }^{6}$ \\ Riccardo Masetti (D) $^{3}$ \\ Arcangelo Prete ${ }^{3}$ \\ Stefano Fanti ${ }^{5}$ \\ Tiziana Lazzarotto ${ }^{4}$
}

'Section of Microbiology, Department of Specialized, Experimental, and Diagnostic Medicine, University of Bologna, Bologna, Italy; ${ }^{2}$ Department of Public Health, Local Health Authority of Bologna, Bologna, Italy; ${ }^{3}$ Pediatric Oncology and Haematology Unit "Lalla Seragnoli", Department of Pediatrics, IRCCS St. Orsola Polyclinic, University of Bologna, Bologna, Italy; ${ }^{4}$ Microbiology Unit, Department of Specialized, Experimental, and Diagnostic Medicine, IRCCS St. Orsola Polyclinic, University of Bologna, Bologna, Italy; ${ }^{5}$ Nuclear Medicine Unit, Department of Specialized, Experimental, and Diagnostic Medicine, IRCCS St. Orsola Polyclinic, University of Bologna, Bologna, Italy; ${ }^{6}$ Microbiology Unit, IRCCS St. Orsola Polyclinic, University of Bologna, Bologna, Italy

Correspondence: Tiziana Lazzarotto Microbiology Unit, Department of Specialized, Experimental, and Diagnostic Medicine, IRCCS St. Orsola Polyclinic,

University of Bologna, via Massarenti 9,

Bologna, 40138 , Italy

Tel +390512143360

Fax +39 0512143514

Email tiziana.lazzarotto@unibo.it

\begin{abstract}
Despite the effectiveness of the currently available antiviral drugs in treating cytomegalovirus (CMV) infection, high rates of adverse effects are associated with their use. Moreover, a problem of increasing importance is the emergence of drug-resistant CMV infection. Here, we describe the first case of off-label use of letermovir (LMV) as preemptive antiviral therapy, in a pediatric allogeneic peripheral blood stem cell transplant recipient with ganciclovir-resistant CMV infection who was intolerant to foscarnet and unable to achieve viral clearance after seven doses of cidofovir. After the administration of LMV, a gradual reduction in viral load was observed and within 6 weeks of LMV treatment, after more than 6 months of positive CMV-DNAemia, the patient cleared the infection. No adverse effects associated with LMV were observed during treatment. In this pediatric study case, the offlabel use of LMV for the treatment of CMV infection has been well tolerated and proved to be effective in leading to the suppression of viral replication.
\end{abstract}

Keywords: pediatric allogeneic peripheral blood stem cell transplant, GCV-resistant CMV, intolerance to FOS, off-label letermovir use

\section{Introduction}

Cytomegalovirus (CMV) reactivation is a major infectious complication after allogeneic hematopoietic stem cell transplant (allo-HSCT). ${ }^{1}$ The incidence of CMV reactivation among the patients at high risk for infection (CMV seronegative donor/ seropositive recipient; $\mathrm{D}-(\mathrm{R}+)$ is about $60-70 \%$ and CMV replication itself has been associated with increased non-relapse mortality. ${ }^{2,3}$ The current strategies for the management of infection consist of the administration of CMV DNA polymerase inhibitors. However, despite their effectiveness in treating CMV infection, high rates of adverse effects are associated with their use. ${ }^{4}$ Moreover, a problem of increasing importance is the emergence of drug-resistant $\mathrm{CMV}$ infection. ${ }^{5}$ In this scenario, a recently developed anti-CMV drug, letermovir (LMV), having a favorable side effect profile and a different viral target (ie, the viral terminase) was approved by the Food and Drug Administration in November 2017 for CMV prophylaxis in adult CMVseropositive allo-HSCT recipients. ${ }^{4,6}$

To the best of our knowledge, here we describe the first case of off-label use of LMV as preemptive antiviral therapy in a pediatric allo-HSCT recipient with ganciclovir (GCV)-resistant CMV infection who was intolerant to foscarnet (FOS) and unable to achieve viral clearance with cidofovir (CDV). 


\section{Description of the Case Report}

The post-transplant course (up to day +670 ) of a 17-year-old male suffering from beta-thalassemia who developed two episodes of acute graft-versus-host-disease (aGVHD) received a diagnosis of early probable EBV disease and experienced an early asymptomatic CMV infection characterized by an incomplete suppression of viral replication. Despite more than a five-month-long administration of CMV standard therapy, it was necessary to administer LMV for 56 days in order to achieve complete viral clearance.

At the Operative Unit of Pediatrics - Oncology, Haematology, and Stem Cell Transplantation Program - of the IRCCS St. Orsola Polyclinic of Bologna, the patient received $\mathrm{CD} 34+$ peripheral blood stem cells at the dose of $9.61 \times 10^{6} / \mathrm{kg}$, from an unrelated mismatched 39-year-old male donor provided by the German Bone Marrow Donor Registry - DKMS Deutsche Knochenmarkspenderdatei $\mathrm{GmbH}$. The conditioning regimen consisted of treosulfan $(14 \mathrm{~g} / \mathrm{mq}$ day -6 to -4$)$, thiotepa $(10 \mathrm{mg} / \mathrm{kg} /$ day on day -7$)$ and fludarabine ( $40 \mathrm{mg} / \mathrm{mq}$ day -6 to -3 ). GVHD prophylaxis consisted of an association of Cyclosporine A (CsA; $3 \mathrm{mg} / \mathrm{kg} / \mathrm{die}$ ) starting on day -1 and short-term methotrexate (on days $+1,+3,+6$ and +11 ). CsA was maintained at a blood concentration ranging from 200 to $300 \mathrm{ng} / \mathrm{mL}$. Acyclovir (ACV) prophylaxis was administered from day -1 to +18 at a dose of $250 \mathrm{mg} / \mathrm{mq}$ three times a day, followed by a $400 \mathrm{mg}$ oral dose, three times a day until anti-CMV therapy was given (day +41). At the time of transplant, CMV serostatus was D-/R+. As previously described, ${ }^{7}$ a post-transplant surveillance of CMV infection was performed on whole blood samples by quantitative real-time PCR assay (CMV ELITe MGB ${ }^{\mathrm{TM}}$ kit; ELITech Group, Italy). Whole blood samples were also investigated for the search of EpsteinBarr virus (EBV)-DNA (EBV ELITe MGB ${ }^{\text {TM }}$ kit; ELITech Group, Italy); ${ }^{8} \mathrm{EBV}$ serostatus was $\mathrm{D}-\mathrm{R}+$. CMV and EBVDNA kinetics, the clinical management of the infections and the immunosuppressive therapies are shown in Figure 1.

Engraftment was achieved with a neutrophil count $>500$ / $\mathrm{mmc}$ at 14 days post-transplant. Three days later (day +17$)$, the patient showed clinical signs related to cutaneous aGVHD (grade II) and, as a consequence, steroids (ie, prednisone [PDN] at $2 \mathrm{mg} / \mathrm{kg} /$ day) were administered for 12 days and then tapered until discontinuation after 15 days. At day +33 , the patient resulted positive for CMV-DNA (954 copies $/ \mathrm{mL}$ ) and when blood viral load was equal to 17,511 copies $/ \mathrm{mL}$ (day +42 ), anti-CMV therapy (iv GCV at a full dose of $5 \mathrm{mg} / \mathrm{kg}$ twice daily, renal function adjusted) was initiated. At day +47 , mycophenolate mofetil (MMF; $750 \mathrm{mg}$ three times a day) for grade II aGVHD (skin and gastrointestinal involvement) was given; MMF was discontinued after 24 days when GVHD manifestations resolved. At day +64, after 3 weeks of antiCMV therapy at full dose, blood viral load was reduced by 1 $\log _{10}$ and iv GCV was administered once daily for the next 2 weeks; no CMV-related symptoms were observed since the

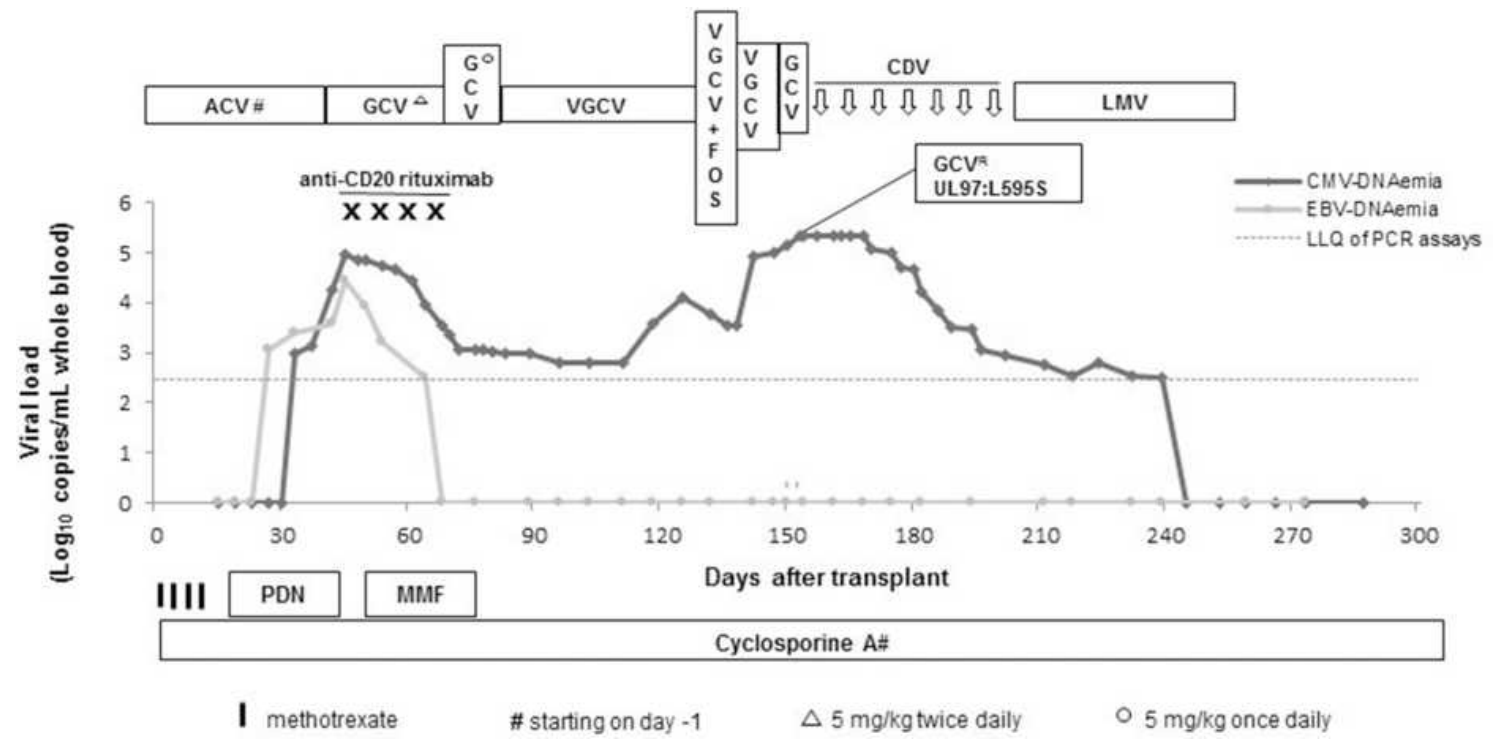

Figure I CMV and EBV-DNA kinetics, infections' clinical management and immunosuppressive treatments. Please refer text for dosages of all drugs. Lower limit of quantification (LLQ) of CMV and EBV PCR assays: $2.4 \log _{10}$ copies $/ \mathrm{mL}$ whole blood.

Abbreviations: ACV, acyclovir; GCV, ganciclovir; VGCV, valganciclovir; FOS, foscarnet; CDV, cidofovir; LMV, letermovir; R, resistant; PDN, prednisone; MMF, mycophenolate mofetil. 
onset of CMV infection. At day +78 , the patient was discharged from the hospital in stable conditions, CMVDNAemia was 1099 copies/mL and valganciclovir (VGCV) treatment at a dosage of $450 \mathrm{mg}$ twice daily (renal function adjusted) was initiated. During the next 4 weeks, CMV-DNA levels decreased up to 620 copies/mL (median: 615 copies/ $\mathrm{mL}$ ). However, at day +125 a CMV-DNA value of 12,925 copies/mL was detected, the patient was hospitalized and FOS was initiated $(90 \mathrm{mg} / \mathrm{kg}$ every 12 hours, renal function adjusted; iv hydration prior to each dose of foscarnet was administered). Adverse effects such as paresthesia in arms and legs, asthenia, pre-cordial pain, agitated and confusional state were observed after FOS doses, therefore antiviral drug dosing was decreased to $60 \mathrm{mg} / \mathrm{kg}$ every 8 hours. Despite this, the adverse effects continued leading to treatment discontinuation after 5 days; hereafter a complete resolution of the symptoms was observed. During the next days, a sudden increase of viral load was observed underuse of VGCV alone (450 mg twice daily, renal function adjusted) and then of iv GCV ( $5 \mathrm{mg} / \mathrm{kg}$ twice daily, renal function adjusted). Consequently, at day +153 antiviral treatment was changed to CDV (5 mg/ $\mathrm{kg} /$ week), and the genotypic resistance testing by Sanger sequencing of PCR-amplified UL97 and UL54 gene segments was performed showing mutant CMV strain (L595S, UL97 gene). ${ }^{9,10}$ Since high levels of CMV-DNAemia (median: 214,174 copies/mL; range: 47,687-223,602) persisted throughout four weeks of treatment with CDV, a request for off-label use of LMV was submitted to the IRCCS St. Orsola Polyclinic of Bologna Drug-Ethics Committee (day +182$)$. While waiting for the Institution's replay, another three doses of CDV were administered and the levels of CMV-DNAemia decreased to 923 copies/mL (day +202). CDV was well tolerated and no worsening of renal function was observed during the preemptive treatment period (serum creatinine values < $0.7 \mathrm{mg} / \mathrm{dL}$ ). After the achievement of the favorable opinion from the Drug-Ethics Committee, the patient was discharged on oral LMV treatment at a dose of $240 \mathrm{mg}$ /day given that it was co-administered with CsA (day +203). One week later, the patient showed signs compatible with peripheral paralysis of the seventh cranial nerve. The patient achieved CMV-DNA negativity within 42 days of LMV treatment. Since LMV inhibits new virion formation, ${ }^{11}$ the shell vial culture for the detection of CMV viremia was performed during the duration of antiviral therapy and resulted negative at each visit. ${ }^{12}$ LMV was discontinued at day +259 ; in the meantime, the resolution of the peripheral paralysis of the seventh cranial nerve was observed. From here onwards, no other episode of CMV infection occurred.

Simultaneously to CMV infection (day +27), the patient resulted positive for EBV-DNA and two days later, given that EBV-DNAemia was equal to 28,416 copies/mL and patient presented fever and significant lymphadenopathy, treatment with anti-CD20 monoclonal antibody rituximab (375 mg/m2/week) was started. At day +51 , whole body ${ }^{18}$ F-fluoro-2-deoxy-D-glucose ( ${ }^{18} \mathrm{~F}$-FDG)-positron emission tomography-computed tomography $(\mathrm{PET} / \mathrm{CT})$ was performed and showed positive uptake in multiple supra and subdiaphragmatic lymph nodes in right and left laterocervical region, right and left axillary region, right pulmonary hilum and, together with abdominal, right iliac and inguinal regions (Figure 2A). Based on patients' clinical status and ${ }^{18}$ F-FDG PET/CT imaging, a probable EBV disease was diagnosed. ${ }^{13}$ At day +67 , after a total of 4 administrations of anti-CD20 therapy, the patient achieved EBV-DNA negativity and a further ${ }^{18} \mathrm{~F}$-FDG $\mathrm{PET} / \mathrm{CT}$ scan performed one month later the diagnosis of probable EBV disease, showed a complete disease remission (Figure 2B). No relapse of EBV infection was observed during the remaining posttransplant period.

\section{Discussion}

Findings from a Phase III, multicenter, double-blind, placebo-controlled, randomized, prospective clinical trial for the evaluation of the safety and efficacy of LMV for CMV prophylaxis in adult CMV-seropositive allo-HSCT recipients (without detectable CMV-DNA at randomization) by Marty and colleagues showed that the administration of LMV reduced the risk of clinically significant CMV infection and the all-cause mortality through week 24 post-transplant. ${ }^{14}$ Furthermore, a subsequent analysis on this trial population performed by Ljungman et al showed that the patients receiving LMV had a lower risk for all-cause mortality than patients receiving placebo also at 48 weeks after HSCT. ${ }^{15}$ Finally, similar outcomes in terms of lower rates of both clinically significant CMV infection and all-cause mortality were observed by Marty et al in patients who received LMV with detectable CMV-DNA at randomization (median value, 150 copies/mL plasma [range, 150-716 LMV; range 150-253 placebo]) compared with those without detectable CMV-DNA at randomization. ${ }^{16}$

With regard to the currently available literature on the efficacy of off-label use of LMV for both the treatment 

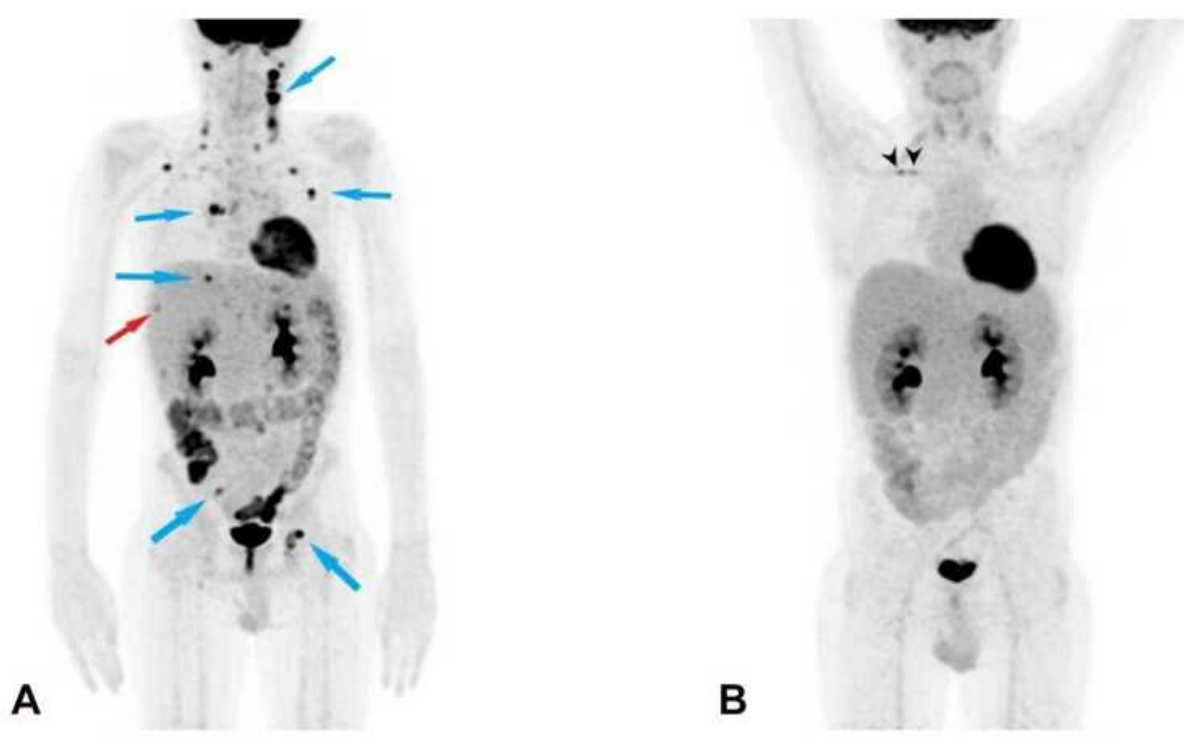

Figure 2 Baseline (at diagnosis) ${ }^{18} \mathrm{~F}$-FDG PET/CT (A) and post-treatment ${ }^{18} \mathrm{~F}$-FDG PET/CT (B). (A) ${ }^{18} \mathrm{~F}$-FDG PET/CT shows pathological uptake in the following lymph nodes (blue arrows): bilateral laterocervical (SUVmax 21.3 at left station III), bilateral axillary (SUVmax 9.8 in left axilla), right costophrenic recess (SUVmax 6.6), right pulmonary hilum (SUVmax II.5), celiac, left paraaortic (SUVmax 4.7), right iliac (SUVmax 5.5), bilateral inguinal (SUVmax II.8 left node) and a pathological uptake at VIs/VIls liver segment (SUVmax 3.3, red arrow). (B) Post-treatment ${ }^{18}$ F-FDG PET/CT shows a complete metabolic response (no pathological uptake); two foci uptake can be seen at central venous catheter in right subclavian vein, suspicious for infection (black arrowheads).

and the secondary prophylaxis of CMV infection after solid organ and hematopoietic stem cell transplant, it is scarce and mostly limited to case reports. ${ }^{6,17-24}$

Here, we describe a case of successful off-label use of LMV as pre-emptive therapy for GCV-resistant CMV infection in a pediatric allo-HSCT recipient who was unable to clear the infection after treatment with the other standard anti-CMV drugs currently available. Risk factors for drug-resistant CMV infection were reverse D-/ $\mathrm{R}+$ mismatch, unrelated mismatched donor and episodes of aGVHD ( $<100$ days post-HSCT). As expected, CMV reactivation occurred in the early post-transplant period $(<$ 100 days post-HSCT) and according to literature data reporting that GVHD and its treatment represent a risk for CMV replication, ${ }^{25,26}$ it was preceded by an episode of aGVHD. Furthermore, evidence for a bidirectional relationship between CMV replication and aGVHD has been reported and the patient developed a second episode of aGVHD during the first weeks of active viral replication. ${ }^{26}$ Simultaneously to CMV reactivation, the patient developed a symptomatic EBV infection. An early $(<1$ year post-transplant) probable EBV disease was diagnosed and was effectively treated by administering anti-CD20 therapy, confirming the literature data. ${ }^{13,27}$

After the first course of antiviral therapy with GCV at standard dose followed by GCV at maintenance dose, the patient did not achieve CMV-DNA negativity. No viral genotypic resistance testing was performed due to the low levels of CMV-DNAemia (ie, < 1200 copies/mL). ${ }^{9}$ When the subsequent combined GCV - FOS therapy was administered, an intolerance to FOS was observed. Soon after, when CMV antiviral resistance was suspected, viral genotypic analysis revealed one of the most important canonical UL97 mutations, ie, L595S, that confer a 9.2-fold resistance to $\mathrm{GCV}^{28,29}$ It is known that a risk factor for the emergence of drug-resistance is a prolonged anti-CMV drug exposure with ongoing replication. ${ }^{5}$ The only other available standard antiCMV drug among those approved for CMV treatment was CDV, and given that is known that it is nephrotoxic, the incomplete suppression of viral replication after a long drug exposure led to the off-label use of LMV as preemptive anti-CMV therapy. After the LMV administration, a gradual reduction in viral load was observed proving the efficacy of the drug. Within 6 weeks of LMV treatment, after more than 6 months of positive CMVDNAemia, the patient achieved CMV-DNA negativity and no infectious virions were isolated from patient blood during LMV treatment. The patient did not show adverse effects associated with LMV and, as often happens, the etiology of the peripheral paralysis of the seventh cranial nerve remained unknown. At the time 
of writing, the patient is alive and well and remained CMV-infection free. ${ }^{30}$

\section{Conclusion}

In this pediatric study case, the off-label use of LMV as preemptive anti-CMV treatment has been well tolerated and proved to be effective in leading to the resolution of CMV infection. Of note, we are aware that in vitro studies report that LMV has a relatively low genetic barrier to resistance and generally support at least an additive effect (if not a synergistic one) of combining LMV with DNA polymerase inhibitors. ${ }^{31}$ However, in our patient, due to GCV-resistance, intolerance to FOS and risk of CDV-related side effects, the use of LMV-based combination therapy was not possible and LMV was administrated as monotherapy.

Many real-world data need to be collected to adequately support the use of LMV as salvage therapy for refractory/resistant CMV infection mostly associated with poor outcome and/or as an alternative in case of adverse effects due to standard anti-CMV drugs. In particular, regarding LMV, appropriate dosage for treatment of CMV infection or disease, clinical efficacy, safety, the in vivo evolution of LMV-resistant CMV as well as the rate of resistance compared with that of other antiviral drugs need to be evaluated in the near future.

\section{Ethics and Consent Statement}

The approval for off-label use of letermovir was obtained by the IRCCS St. Orsola Polyclinic of Bologna DrugEthics Committee (Prot. n. 19149/CF AVEC, on May 20, 2019). Written informed consent was obtained from the patient, having come of age during our monitoring, for the publication of this case report and the accompanying image. Institutional approval was not required to publish the case details.

\section{Acknowledgments}

This research did not receive any specific grant from funding agencies in the public, commercial, or not-forprofit sectors. The authors would like to thank Dr. Marta Leone for contributing to data collection and their Linguistic Consultant, Lucy Scioscia, for editing the English language text.

\section{Disclosure}

The authors declare no conflicts of interest for this work.

\section{References}

1. Stern L, Withers B, Avdic S, et al. Human cytomegalovirus latency and reactivation in allogeneic hematopoietic stem cell transplant recipients. Front Microbiol. 2019;10:1186. doi:10.3389/fmicb.2019.01186

2. Ruell J, Barnes C, Mutton K, et al. Active CMV disease does not always correlate with viral load detection. Bone Marrow Transplant. 2007;40:55-61. doi:10.1038/sj.bmt.1705671

3. Ljungman P, de la Camara R, Robin C, et al.; on behalf of the 2017 European Conference on Infections in Leukaemia group. Guidelines for the management of cytomegalovirus infection in patients with haematological malignancies and after stem cell transplantation from the 2017 European Conference on Infections in Leukaemia (ECIL 7). Lancet Infect Dis. 2019;19:e260-e272. doi:10.1016/S1473-3099(19)30107-0

4. El Helou G, Razonable RR. Letermovir for the prevention of cytomegalovirus infection and disease in transplant recipients: an evidence-based review. Infect Drug Resist. 2019;12:1481-1491. doi:10.2147/IDR.S180908

5. Chemaly RF, Chou S, Einsele H, et al. Definitions of resistant and refractory cytomegalovirus infection and disease in transplant recipients for use in clinical trials. Clin Infect Dis. 2019;68:1420-1426. doi:10.1093/cid/ciy696

6. Phoompoung P, Ferreira VH, Tikkanen J, et al. Letermovir as salvage therapy for cytomegalovirus infection in transplant recipients. Transplantation. 2020;104:404-409. doi:10.1128/AAC.02337-18

7. Chiereghin A, Potena L, Borgese L, et al. Monitoring of cytomegalovirus (CMV)-specific cell-mediated immunity in heart transplant recipients: clinical utility of the QuantiFERON-CMV assay for management of posttransplant CMV infection. J Clin Microbiol. 2018;56: e01040-17. doi:10.1128/JCM.01040-17

8. Chiereghin A, Piccirilli G, Belotti T, et al. Clinical utility of measuring Epstein-Barr virus specific cell mediated immunity after HSCT in addition to virological monitoring: results from a prospective study. Med Microbiol Immunol. 2019;208:825-834. doi:10.1007/s00430-01900629-2

9. Allice T, Busca A, Locatelli F, Falda M, Pittaluga F, Ghisetti V. Valganciclovir as preemptive therapy for cytomegalovirus infection post allogenic stem cell transplantation: implications for the emergence of drug-resistant cytomegalovirus. J Antimicrob Chemother. 2009;63:600-608. doi:10.1093/jac/dkn521

10. Lurain NS, Chou S. Antiviral drug resistance of human cytomegalovirus. Clin Microbiol Rev. 2010;23:689-712. doi:10.1128/CMR.00009-10

11. Gentry BG, Bogner E, Drach JC. Targeting the terminase: an important step forward in the treatment and prophylaxis of human cytomegalovirus infections. Antiviral Res. 2019;161:116-124. doi:10.1016/j. antiviral.2018.11.005

12. Cassaniti I, Colombo AA, Bernasconi P, et al. Positive HCMV DNAemia in stem cell recipients undergoing letermovir prophylaxis is expression of abortive infection. Am J Transplant. 2021:1-7. doi:10.1111/ajt.16450.

13. Styczynski J, van der Velden W, Fox CP, et al.; Sixth European Conference on Infections in Leukemia, a joint venture of the Infectious Diseases Working Party of the European Society of Blood and Marrow Transplantation (EBMT-IDWP), the Infectious Diseases Group of the European Organization for Research and Treatment of Cancer (EORTC-IDG), the International Immunocompromised Host Society (ICHS) and the European Leukemia Net (ELN). Management of Epstein-Barr Virus infections and post-transplant lymphoproliferative disorders in patients after allogeneic hematopoietic stem cell transplantation: sixth European Conference on Infections in Leukemia (ECIL-6) guidelines. Haematologica. 2016;101:803-811. doi:10.3324/ haematol.2016.144428

14. Marty FM, Ljungman P, Chemaly RF, et al. Letermovir prophylaxis for cytomegalovirus in hematopoietic-cell transplantation. $N$ Engl J Med. 2017;377:2433-2444. doi:10.1056/nejmoa1706640 
15. Ljungman P, Schmitt M, Marty FM, et al. A mortality analysis of letermovir prophylaxis for cytomegalovirus (CMV) in CMV-seropositive recipients of allogeneic hematopoietic cell transplantation. Clin Infect Dis. 2020;70:1525-1533. doi:10.1093/cid/ciz490

16. Marty FM, Ljungman PT, Chemaly RF, et al. Outcomes of patients with detectable CMV DNA at randomization in the Phase III trial of letermovir for the prevention of CMV infection in allogeneic hematopoietic cell transplantation. Am J Transplant. 2020;20:1703-1711. doi:10.1111/ajt.15764

17. Cherrier L, Nasar A, Goodlet KJ, Nailor MD, Tokman S, Chou S. Emergence of letermovir resistance in a lung transplant recipient with ganciclovir-resistant cytomegalovirus infection. Am J Transplant. 2018;18:3060-3064. doi:10.1111/ajt.15135

18. Koepf US, Klehr HU, Eis-Huebinger AM, et al. Suppression of CMV infection with letermovir in a kidney transplant patient. Eur J Case Rep Intern Med. 2020;7:001622. doi:10.12890/2020 001622

19. Frietsch JJ, Michel D, Stamminger T, et al. In vivo emergence of UL56 C325Y cytomegalovirus resistance to letermovir in a patient with acute myeloid leukemia after hematopoietic cell transplantation. Mediterr J Hematol Infect Dis. 2019;11:e2019001. doi:10.4084/ MJHID.2019.001

20. Kaul DR, Stoelben S, Cober E, et al. First report of successful treatment of multidrug-resistant cytomegalovirus disease with the novel anti-CMV compound AIC246. Am $J$ Transplant. 2011;11:1079-1084. doi:10.1111/j.1600-6143.2011.03530.x

21. Kilgore JT, Becken B, Varga MG, et al. Use of letermovir for salvage therapy for resistant cytomegalovirus in a pediatric hematopoietic stem cell transplant recipient. $J$ Pediatric Infect Dis Soc. 2020;9:486-489. doi:10.1093/jpids/piz050

22. Turner N, Strand A, Grewal DS, et al. Use of letermovir as salvage therapy for drug-resistant cytomegalovirus retinitis. Antimicrob Agents Chemother. 2019;63:e02337-18. doi:10.1128/AAC.02337-18

23. Lin A, Maloy M, Su Y, et al. Letermovir for primary and secondary cytomegalovirus prevention in allogeneic hematopoietic cell transplant recipients: real-world experience. Transpl Infect Dis. 2019;21: e13187. doi:10.1111/tid.13187
24. Robin C, Thiebaut A, Alain S, et al. Letermovir for secondary prophylaxis of CMV infection and disease after allogeneic hematopoietic cell transplantation: results from the French compassionate program. Biol Blood Marrow Transplant. 2020;26(5):978-984. doi:10.1016/j.bbmt.2020.01.027

25. Sousa H, Boutolleau D, Ribeiro J, et al. Cytomegalovirus infection in patients who underwent allogeneic hematopoietic stem cell transplantation in Portugal: a five-year retrospective review. Biol Blood Marrow Transplant. 2014;20:1958e1967. doi:10.1016/j. bbmt.2014.08.010

26. Cantoni N, Hirsch HH, Khanna N, et al. Evidence for a bidirectional relationship between cytomegalovirus replication and acute Graft-versus-Host Disease. Biol Blood Marrow Transplant. 2010;16:1309-1314. doi:10.1016/j.bbmt.2010.03.020

27. Chiereghin A, Bertuzzi C, Piccirilli G, et al. Successful management of EBV-PTLD in allogeneic bone marrow transplant recipient by virological-immunological monitoring of EBV infection, prompt diagnosis and early treatment. Transpl Immunol. 2016;34:60-64. doi:10.1016/j.trim.2015.12.002

28. Campos AB, Ribeiro J, Boutolleau D, Sousa H. Human cytomegalovirus antiviral drug resistance in hematopoietic stem cell transplantation: current state of the art. Rev Med Virol. 2016;26:161-182. doi:10.1002/rmv.1873

29. Göhring K, Hamprecht K, Jahn G. Antiviral drug- and multidrug resistance in cytomegalovirus infected SCT patients. Comput Struct Biotechnol J. 2015;13:153-159. doi:10.1016/j.csbj.2015.01.003

30. Ciorba A, Corazzi V, Conz V, Bianchini C, Aimoni C. Facial nerve paralysis in children. World J Clin Cases. 2015;3:973-979. doi:10.12998/wjcc.v3.i12.973

31. Hakki M. Moving past ganciclovir and foscarnet: advances in CMV therapy. Curr Hematol Malig Rep. 2020;15:90-102. doi:10.1007/ s11899-020-00557-6
Infection and Drug Resistance

\section{Publish your work in this journal}

Infection and Drug Resistance is an international, peer-reviewed openaccess journal that focuses on the optimal treatment of infection (bacterial, fungal and viral) and the development and institution of preventive strategies to minimize the development and spread of resistance. The journal is specifically concerned with the epidemiology of

\section{Dovepress}

antibiotic resistance and the mechanisms of resistance development and diffusion in both hospitals and the community. The manuscript management system is completely online and includes a very quick and fair peerreview system, which is all easy to use. Visit http://www.dovepress.com/ testimonials.php to read real quotes from published authors. 